\title{
Study of Genetic Divergence in Advance Breeding Lines of Soybean [Glycine max (L.) Merrill] for Yield Attributing Traits
}

\author{
Stuti Mishra*, Avinash Jha, D. K. Panchshwar and A. N. Shrivastava
}

Dept. of Plant Breeding \& Genetics, Jawaharlal Nehru Krishi Vishwa Vidyalya, Jabalpur, Madhya Pradesh (482 004), India

\section{Corresponding Author}

Stuti Mishra

e-mail: stuti.curious@gmail.com
Article History

Article ID: 3 C0422

Received in $07^{\text {th }}$ October, 2017

Received in revised form $21^{\text {st }}$ December, 2017

Accepted in final form $29^{\text {th }}$ January, 2018

\begin{abstract}
An investigation was conducted during Kharif 2015 with sixty advances breeding lines of soybean, to identify the most diverse and promising lines for hybridization through genetic diversity analysis by Mahalanobis's $D^{2}$ statistics. The $D^{2}$ statistics is a powerful tool to measure genetic divergence within a population and to estimate the contribution of each trait to the total diversity. In present study observations on twelve yield attributing traits were recorded on lines raised with 3 replications in RBD. The results revealed significant differences among lines for all studied traits. Sixty soybean lines were grouped in 16 clusters following Tocher's method. Cluster I was polygenotypic (14 lines) followed by cluster III (12 lines), cluster V (8 lines), cluster VIII (7 lines), XV (8 lines), while remaining 11 clusters were monogenotypic. Entries from cluster I, III, V, VIII, IX, XIII and XV can be used in hybridization programme for development of resistant and high yielding lines. The identified potential lines based on the $D^{2}$ statistics were JS 20-49, JS 20-109, JS 20-116, JS 20-94, JS 21-03, JS 21-04, JS 20-79, JS 20-87, JS 20-89, JS 2069, JS 20-98, JS 20-34, JS 20-29, JS 20-53, NRC 37, RVS 2007-1, JSM 242, JS 335, JSM 230, JS 20-86. Among twelve traits, numbers of pods plant $^{-1}$, number of seeds plant ${ }^{-1}$ and seed yield plant ${ }^{-1}$ were maximum contributing traits to genetic diversity.
\end{abstract}

Keywords: Soybean breeding lines, $\mathrm{D}^{2}$ statistics, genetic diversity

\section{Introduction}

Soybean [Glycine max (L.) Merill] is the chief oilseed crop in India contributes $43 \%$ oilseeds and $29 \%$ edible oil production in the country. The contribution of Madhya Pradesh has always been largest and extensive in terms of area and production of country's total. The yield level of soybean reduced drastically due to impact of abiotic and biotic stress and lack of appropriate breeding strategies. In the year 2014-15 the estimate of soybean area, production and productivity in India was 108 lakh ha, 87.10 lakh tons and $800 \mathrm{~kg} \mathrm{ha}^{-1}$ and in M.P. area reached to 55.46 lakh ha, production 49.67 lakh tons, and productivity of $815 \mathrm{~kg} \mathrm{ha}^{-1}$. The yield level in soybean is hovering around $3.0 \mathrm{t} \mathrm{ha}^{-1}$ which is moderately low. Large yield gap requires superior pre-breeding efforts to broaden the genetic base suitable for screening better lines. The year to year fluctuations in production and productivity are mainly accounted to erratic behavior of monsoon for instance in 2013, the whole soybean belt received heaviest rainfall ( 2400 $\mathrm{mm}$ ) that resulted in severe water logging and high moisture conditions resulting huge reduction in yield of the crop. On the contrary in the year 2014 and again in present year 2015 soybean crop received very scarce rains and high temperature during crop season caused low yields. Analysis of genetic diversity is foremost important step for any crop improvement programme. In belief of Shrivastava et al. (2011), there is a possibility of increasing soybean yield potential upto $27 \%$ through developing four seeded plant ideotype. Information of diversity among population is useful to categorize germplasm for identification of promising sources to aid in selection of parents for hybridization. Genetically diverse parent is a foremost requirement to enhance the selection of superior segregants for abiotic stresses and various traits. When such parents utilized in cross breeding programme, they are likely toproduce high heterotic effect and wide spectrum of variability (Sichkar et al., 1988; Barh et al., 2014). Soybean being a self-pollinated oilseed crop faces the constraint of narrow genetic base.

Thus, present study aims to analyze the genetic diversity of sixty advance breeding lines of soybean for identifying high yielding promising parental lines which can withstand moisture stress conditions (high and low). This study is especially important for central region of India, where moisture stress low or high, one or the other year in rainy season changes during crop growth period. Selection of genotypes with wider adaptability and enhanced genetic diversity is key factor for development of varieties. The Mahalanobis's $D^{2}$ statistics is a powerful tool to measure genetic divergence within a set 
of genotypes. It allows accurate comparison among all the population in given group and degree of diversity among them. The genotypes which have greater morphological similarity were grouped in clusters. (Ghatgeand and Kadu, 1993). Das et al. (2001) reported that grouping pattern of the diverse genotypes suggested no parallelism between genetic divergence and geographical distribution of the genotypes.

\section{Materials and Methods}

The investigation was carried out consisting of sixty advance breeding lines of soybean which includes mutant lines, released varieties, and advance breeding generations. The experiment was conducted during Kharif, 2015 in RBD with three replications at Seed Breeding Farm, JNKVV, Jabalpur; M.P. Observations were recorded for phenological traits and ten morphological, traits. Five random plants were selected to record data on phenological traits i.e. vegetative and reproductive stages and morphological traits viz., plant height per plant, number of branches plant ${ }^{-1}$, number of nodes plant $^{-1}$, number of pods plant ${ }^{-1}$, number of pod cluster plant ${ }^{-1}$, number of seeds plant ${ }^{-1}, 100$ seed weight, biological yield, harvest index and seed yield plant ${ }^{-1}$. The replicated data of phenological and quantitative traits were subjected to genetic divergence analysis using Mahalanobis's D2 (Mahalanobis, 1936) statistic as suggested by Rao (1952). All the soybean lines were grouped into respective clusters on the basis of values following Toucher's method.

After testing the difference between genotypes for each of the trait, a simultaneously test of significance for differences in the mean value of number of correlated variables with regard to the pooled effect of 12 traits was carried out using ' $V$ ' statistic which in turn utilizes Wilk's ' $\wedge$ ' criterion. The sums of squares and sums of products of error and error+variety were used for this purpose. The estimation of ' $\Lambda$ ' (Wilk's criterion) was done using the following relationship.

' $\Lambda$ '=(E)/ (E+V)

Where,

$(E)=$ Determination of error sum of squares and sum of products matrix.

$(E+V)=$ Determination of error + Varieties sum of squares a sum of products matrix.

\section{Results and Discussion}

\subsection{Mahalanobis generalized distance $\left(D^{2}\right)$ :}

For the determination of genetic divergence among sixty parentages of soybean Wilk's $\Lambda$ criterion value (15291.477) was found highly significant. The $V$ statistics value (3128.726) was also highly significant at 588 degree of freedom. It showed that genotypes differed significantly, when all the traits were considered simultaneously. The analysis of variance showed highly significant differences within the population for most of the traits studied. The $D^{2}$ values corresponding to possible comparison among sixty advance breeding lines were computed separately in the analysis.

\subsection{Contribution of individual trait towards genetic divergence}

The percentage contribution towards genetic divergence by all the traits is presented in Table 1 . The traits viz., number of pods per plant (30.04\%) (Adsul and Monpara, 2014) (Kayande et al., 2009; Dhapke et al. 2011) and biological yield plant ${ }^{-1}$ (29.40\%) contributed most towards genetic divergence followed by remaining traits in descending order viz.seed yield plant ${ }^{-1}(14.62 \%)$, number of seeds plant ${ }^{-1}(7.23 \%)$, (Tyagi et al., 2012) (Jency and Kalaimagal, 2014) vegetative stage(4.60\%),100 seed weight (g) (4.56\%), number of pod cluster per plant $(4.10 \%)$, reproductive stage (3.17\%) harvest index (1.32\%), and while 3traits viz., plant height $(0.85 \%)$, number of branches per plant $(0.08 \%)$ and number of nodes plant $^{-1}$ contributed less than $1 \%$ towards genetic divergence.

Table 1: Percentage contribution towards genetic divergence by all the traits

\begin{tabular}{|c|c|c|}
\hline Traits & Times ranked & $\begin{array}{l}\text { Percentage } \\
\text { contribution }\end{array}$ \\
\hline No. of pods plant ${ }^{-1}$ & 376 & $30.04 \%$ \\
\hline Biological yield plant $^{-1}(\mathrm{~g})$ & 355 & $29.40 \%$ \\
\hline Seed yield plant ${ }^{-1}$ & 190 & $14.62 \%$ \\
\hline Number of seeds plant ${ }^{-1}$ & 89 & $7.23 \%$ \\
\hline Vegetative stage (days) & 62 & $4.60 \%$ \\
\hline 100 seed weight (g) & 51 & $4.56 \%$ \\
\hline No. of pod clusters plant ${ }^{-1}$ & 43 & $4.10 \%$ \\
\hline Reproductive stage (days) & 30 & $3.17 \%$ \\
\hline Harvest index (\%) & 18 & $1.32 \%$ \\
\hline Plant height $(\mathrm{cm})$ & 9 & $0.85 \%$ \\
\hline No. of branches plant ${ }^{-1}$ & 1 & $0.11 \%$ \\
\hline No. of nodes plant ${ }^{-1}$ & 0 & $0 \%$ \\
\hline
\end{tabular}

\subsection{Grouping of genotypes into different clusters}

On the basis of $D^{2}$ values, the sixty advance breeding lines were grouped into 16 clusters following Tocher's method (Table 2.). Cluster I was polygenotypic (14 lines) followed by cluster III (12 lines), cluster V (8 lines), cluster VIII (7 lines) and cluster XV (8 lines) while remaining 10 clusters were monogenotypic. (Mehetre et al., 1994 and lqbal et al., 2008).

\subsection{Intra and inter cluster divergence $D^{2}$ values}

The average intra and inter-cluster $D^{2}$ values estimated as per the procedure given by Singh and Choudhary (1979) are presented in Table 3 . and the cluster mean values are summarized in Table 4. Cluster VIII showed maximum intra cluster $D^{2}$ value (95.02) followed with cluster XV (72.53), cluster I (72.18) and cluster III (63.85) and cluster V (58.23), 


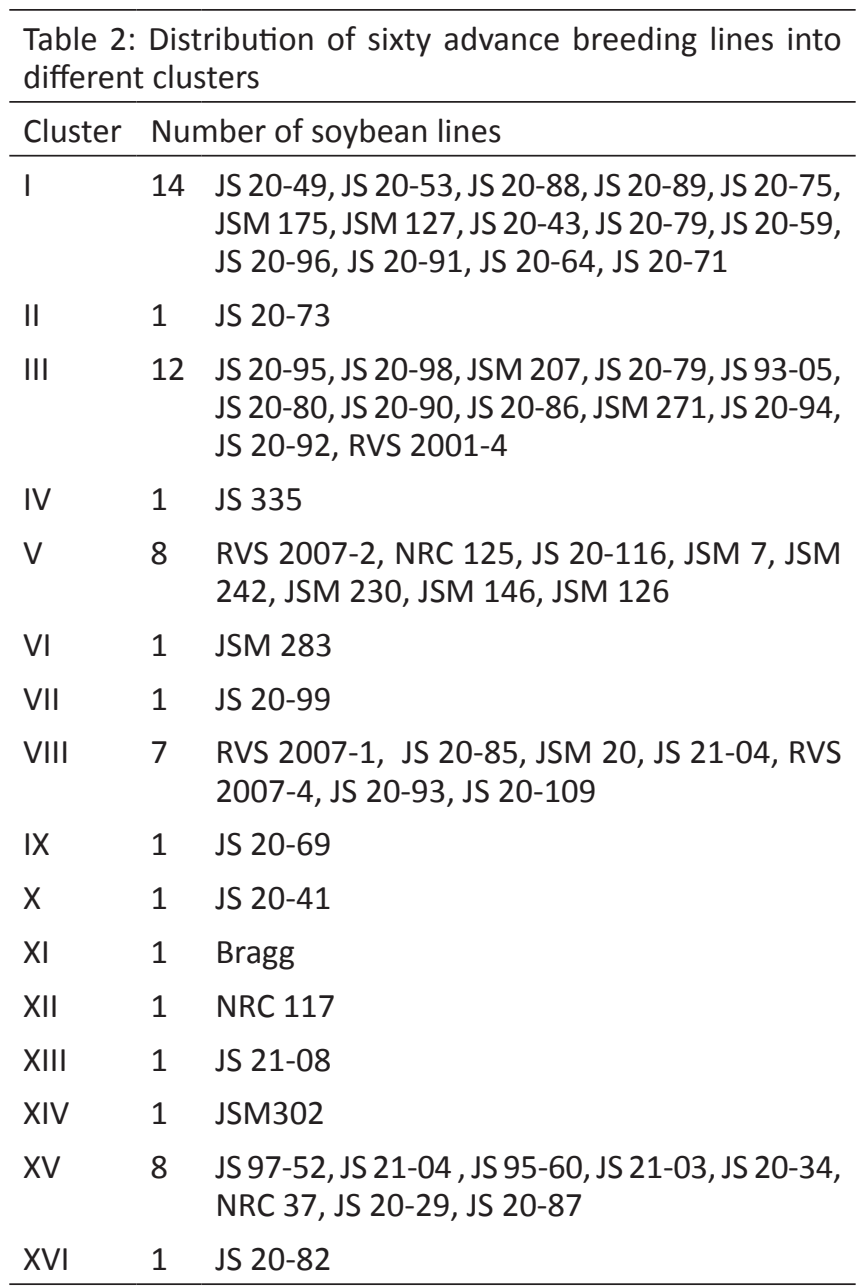

while remaining 10 Clusters were mono-genotypic hence, showed no intra cluster divergence.

The highest inter cluster divergence was observed between genotypes of cluster X and XIII (969.45), followed by cluster IV and X (767.43), XIII and XV (764.66), XIII and XVI (753.37) and $X$ and XII (699.37).

Cluster I (72.18) comprises of 14 soybean lines and was nearest to cluster II (91.25), while cluster XIII (324.13) was farthest or more distantly related to cluster I.

Cluster II (0.00) was mono-genotypic, but nearest to cluster VII (47.74) and placed at maximum distance to cluster XIII (446.2). Cluster III (63.85) was poly-genotypic, but nearest to cluster IV (116.65) and farthest distance to cluster X (593.32).

Cluster IV (0.00) was mono-genotypic, but nearest to cluster XIII (98.74) and was placed at a maximum distance to cluster $X(767.43)$.

Cluster V (58.23) was polygenotypic, but nearest to cluster VI (22.01) however it was placed at a maximum distance to cluster XIII (628.7).

Cluster VI (0.00) was mono-genotypic, but nearest to cluster $X$ (35.39) and placed at maximum distance to cluster XIII (737.63).

Cluster VII (0.00) was mono-genotypic, but nearest to cluster XIV (119.00) and at maximum distance to cluster X (373.98). Cluster VIII (95.02) was poly-genotypic, but nearest to cluster IX (146.17) and found maximum distance to cluster X (503.17). Cluster IX (0.00) was mono-genotypic, but nearest to cluster XII (53.02) and placed at maximum distance to cluster $X$ (668.05). Cluster X (0.00) was mono-genotypic, but nearest to cluster XI (196.06) recorded at maximum distance to cluster XIII (969.45).

Table 3: Inter and Intra Cluster distances for advance breeding lines

\begin{tabular}{|c|c|c|c|c|c|c|c|c|c|}
\hline Clusters & Cluster I & Cluster II & Cluster III & Cluster IV & Cluster V & Cluster VI & Cluster VII & Cluster VIII & Cluster IX \\
\hline Cluster I & 72.18 & 91.25 & 147.03 & 230.31 & 129.30 & 155.78 & 95.10 & 162.57 & 188.51 \\
\hline Cluster II & & 0.00 & 207.91 & 370.21 & 118.19 & 128.99 & 47.74 & 287.58 & 342.15 \\
\hline Cluster III & & & 63.85 & 116.65 & 363.62 & 418.18 & 128.85 & 229.22 & 174.38 \\
\hline Cluster IV & & & & 0.00 & 439.82 & 531.34 & 234.25 & 169.10 & 194.73 \\
\hline Cluster V & & & & & 58.23 & 22.01 & 230.19 & 246.69 & 363.88 \\
\hline Cluster IV & & & & & & 0.00 & 244.69 & 301.40 & 437.65 \\
\hline Cluster VII & & & & & & & 0.00 & 213.66 & 265.88 \\
\hline Cluster VIII & & & & & & & & 95.02 & 146.17 \\
\hline Cluster IX & & & & & & & & & 0.00 \\
\hline \multicolumn{10}{|l|}{ Cluster X } \\
\hline \multicolumn{10}{|l|}{ Cluster XI } \\
\hline \multicolumn{10}{|l|}{ Cluster XII } \\
\hline \multicolumn{10}{|l|}{ Cluster XIII } \\
\hline \multicolumn{10}{|l|}{ Cluster XIV } \\
\hline \multicolumn{10}{|l|}{ Cluster XV } \\
\hline Cluster XVI & & & & & & & & & \\
\hline
\end{tabular}




\begin{tabular}{|c|c|c|c|c|c|c|c|}
\hline Clusters & Cluster X & Cluster XI & Cluster XII & Cluster XIII & Cluster XIV & Cluster XV & Cluster XVI \\
\hline Cluster I & 273.08 & 109.63 & 208.00 & 324.13 & 169.85 & 252.82 & 207.77 \\
\hline Cluster II & 186.86 & 125.35 & 341.62 & 446.20 & 224.26 & 135.25 & 264.60 \\
\hline Cluster III & 593.32 & 234.68 & 145.07 & 133.02 & 158.88 & 431.59 & 487.52 \\
\hline Cluster IV & 767.43 & 385.93 & 203.44 & 98.74 & 118.37 & 611.83 & 529.24 \\
\hline Cluster V & 66.10 & 122.95 & 410.47 & 628.70 & 321.77 & 280.20 & 93.12 \\
\hline Cluster IV & 35.39 & 119.57 & 491.62 & 737.63 & 369.07 & 208.31 & 102.88 \\
\hline Cluster VII & 373.98 & 192.73 & 279.39 & 288.67 & 119.00 & 150.79 & 328.25 \\
\hline Cluster VIII & 503.17 & 277.47 & 229.03 & 310.91 & 155.46 & 437.78 & 197.82 \\
\hline Cluster IX & 668.05 & 257.00 & 53.02 & 263.00 & 292.91 & 606.68 & 307.89 \\
\hline Cluster X & 0.00 & 196.06 & 699.37 & 969.45 & 547.96 & 269.63 & 210.47 \\
\hline Cluster XI & & 0.00 & 221.35 & 541.69 & 347.65 & 255.06 & 218.27 \\
\hline Cluster XII & & & 0.00 & 267.33 & 333.41 & 632.89 & 432.80 \\
\hline Cluster XIII & & & & 0.00 & 151.26 & 764.66 & 753.37 \\
\hline Cluster XIV & & & & & 0.00 & 367.31 & 402.03 \\
\hline Cluster XV & & & & & & 72.53 & 350.73 \\
\hline Cluster XVI & & & & & & & 0.00 \\
\hline
\end{tabular}

\begin{tabular}{lccccccccccccc}
\hline \multicolumn{10}{l}{ Table 4: Cluster means for sixty advance breeding lines } \\
\hline Clusters & VP & RP & PH & NPCP & NPP & NBP & NNP & NPSP & BY & SW & HI & SYP \\
\hline Cluster I & 51.63 & 69.00 & 46.87 & 24.14 & 54.51 & 4.93 & 10.80 & 110.20 & 17.91 & 8.68 & 39.23 & 7.87 \\
Cluster II & 52.33 & 69.00 & 33.67 & 21.53 & 50.73 & 4.97 & 10.27 & 93.00 & 17.58 & 8.90 & 35.33 & 7.47 \\
Cluster III & 45.00 & 65.00 & 35.49 & 24.51 & 53.58 & 5.02 & 10.49 & 106.20 & 21.96 & 7.63 & 31.12 & 7.67 \\
Cluster IV & 51.00 & 70.33 & 33.67 & 17.87 & 30.53 & 5.07 & 8.93 & 59.00 & 12.43 & 6.98 & 35.03 & 4.36 \\
Cluster V & 55.67 & 88.00 & 41.47 & 21.47 & 51.53 & 5.27 & 10.53 & 106.67 & 19.27 & 9.00 & 45.77 & 7.39 \\
Cluster IV & 53.33 & 75.33 & 37.13 & 22.10 & 53.24 & 5.10 & 10.27 & 107.33 & 18.95 & 8.04 & 45.57 & 7.63 \\
Cluster VII & 51.66 & 70.33 & 39.27 & 19.67 & 43.77 & 4.23 & 10.40 & 77.67 & 20.14 & 8.32 & 29.23 & 6.47 \\
Cluster VIII & 44.93 & 69.20 & 31.88 & 17.16 & 50.20 & 4.96 & 10.68 & 90.60 & 13.20 & 7.65 & 44.15 & 7.12 \\
Cluster IX & 50.00 & 84.67 & 33.33 & 16.67 & 38.27 & 3.50 & 8.30 & 68.33 & 13.22 & 7.58 & 42.52 & 6.40 \\
Cluster X & 55.17 & 97.67 & 43.53 & 21.27 & 52.75 & 4.60 & 10.43 & 100.38 & 21.65 & 8.41 & 46.37 & 7.56 \\
Cluster XI & 42.33 & 77.00 & 35.40 & 20.87 & 44.55 & 4.93 & 10.53 & 92.00 & 17.12 & 7.67 & 40.80 & 6.98 \\
Cluster XII & 52.00 & 89.00 & 32.47 & 18.53 & 36.40 & 4.20 & 10.50 & 67.33 & 14.38 & 8.39 & 44.43 & 6.40 \\
Cluster XIII & 43.33 & 67.33 & 41.60 & 11.33 & 26.30 & 2.07 & 10.38 & 50.67 & 14.29 & 8.49 & 30.03 & 4.29 \\
Cluster XIV & 52.33 & 72.33 & 36.77 & 23.80 & 40.10 & 2.60 & 10.23 & 70.00 & 17.74 & 7.19 & 30.40 & 5.38 \\
Cluster XV & 37.67 & 65.00 & 40.89 & 23.33 & 58.67 & 4.07 & 11.00 & 110.33 & 21.00 & 7.70 & 45.98 & 7.59 \\
Cluster XVI & 50.67 & 75.00 & 40.33 & 21.80 & 51.93 & 4.07 & 10.40 & 100.00 & 15.21 & 8.29 & 43.62 & 6.29 \\
\hline
\end{tabular}

VP: Vegetative phase; RP: Reproductive phase; PH: Plant height; NPCP: Num of pod cluster plant ${ }^{-1}$; NPP: Num of pods plant ${ }^{-1}$; NBP: Num of branches plant ${ }^{-1}$; NNP: Num of nodes plant ${ }^{-1}$; NPSP: Num of pod seeds plant ${ }^{-1}$; BY: Biological yield (g); SW: 100 seed weight (g); HI: Harvest index; SYP: Seed yield plant ${ }^{-1}$

Cluster XI (0.00) was mono-genotypic, but nearest to cluster $\mathrm{XVI}$ (218.27) followed by remaining clusters in ascending order viz., cluster XII (221.35), cluster XV (255.06) and cluster XIV (347.65), however it was placed at a maximum distance to cluster XIII (541.69).

Cluster XII (0.00) was mono-genotypic, but nearest to cluster XIII (267.33) followed by remaining clusters in ascending order viz., cluster XIV (333.41) and cluster XVI (432.8), however it 
was placed at a maximum distance to cluster XV (632.89).

Cluster XIII (0.00) was mono-genotypic, but nearest to cluster XIV (151.26) and cluster XVI (753.37), however it was placed at a maximum distance to cluster XV (764.66).

Cluster XIV (0.00) was mono-genotypic and was nearest to cluster XV (367.31) and was placed farthest to cluster XVI (402.03). Cluster XV (72.53) was poly-genotypic and was nearest to cluster XVI (350.03).

Cluster means showing importance of grouped traits are presented in Table 4. Cluster VI, VII, XI, XII, XIV and XVI showed neither high nor low values of traits under study. Cluster $V$ showed highest value for vegetative phase (55.67), cluster XV showed lowest value (37.67). Clusterxrecorded highest value for reproductive phase (98.67) shows that the lines in this cluster can be use to develop moderate to late maturing parents and cluster XV showed lowest value (65.00). Cluster I recorded highest value only for plant height (46.87) and cluster VIII showed lowest value of the trait (31.88). Cluster V recorded highest value for number of branches plant ${ }^{-1}$ (5.27). Cluster III recorded higher value for number of pod cluster plant $^{-1}$ (24.51). Cluster XV recorded highest value for number of pods plant ${ }^{-1}$ (58.67) followed by number of nodes plant ${ }^{-1}$ (11.00), number of seeds plant ${ }^{-1}$ (110.33) and harvest index (45.98). Cluster $V$ showed highest value for 100 seed weight (9.00). Cluster III recorded highest value for biological yield plant $^{-1}$ (21.96). Cluster I recorded highest value for seed yield plant $^{-1}(7.87)$.

\section{Conclusion}

The potential genotypes based on the $D^{2}$ statistics were JS 20-49, JS 20-109, JS 20-116, JS 20-94, JS 21-03, JS 21-04, JS 20-79, JS 20-87, JS 20-89, JS 20-69, JS 20-98, JS 20-34, JS 2029, JS 20-53, NRC 37, RVS 2007-1, JSM 242, JS 335, JSM 230, JS 20-86. These lines belonging to distant clusters may be used for hybridization programme to exploit the heterotic effect and desirable genes to achieve potential segregants.

\section{References}

Adsul, H.R., Mompara, B.A., 2014. Genetic Divergence Analysis in Indigenously Developed Indian Soybean [Glycine max (L.) Merrill] germplasm. Genetika 46(2), 401-409.

Bekele, A., Alema, G.W., Zeleke, H., 2012. Genetic divergence among soybean introductions in Ethiopia based on agronomic traits. Journal of Biology, Agriculture and Healthcare 2(6), 6-12.

Dhapke, S.K., Patil, S.P., Wankhade, R.S., 2011. Studies on genetic diversity in elite soybean genotypes. Crop Research 42(1), 255-258.

Ganeshmurthy, K., Sheshaduri, P., 2002. Genetic divergence in soybean [Glycine max (L.) Merrill]. Madras Agriculture Research Journal 54, 242-246.

Ghatge, R.D., Kadu, R.N., 1993. Genetic diversity in soybean. Annals of Agricultural Research 14(2), 143-148.

Iqbal, Z., Arshad, M., Ashraf, M., Mahmood, T., Waheed, A., 2008. Evaluation of soybean [Glycine max (L.) Merrill] germplasm for some important morphological traits using multivariate analysis. Pakistan Journal of Botany 40(6), 2323-2328.

Jency, P., Kalaimagal, T., 2014. Genetic divergence and association analysis in Soybean. Soybean Research (Special Issue No.2), 81-92.

Kayande, N.V., Patil, S.P., 2009. Genetic divergence in soybean [Glycine max (L.) Merril]. International Journal of Plant Sciences 4(1), 218-222.

Kumar, M., Nadarajan, N., 1994. Genetic divergence studies in soybean [Glycinemax (L.) Merrill]. Indian Journal of Genetics and Plant Breeding 54(3), 242-246.

Mahalanobis, P.C., 1936. On generalized distance in statistics. Proceedings of National Academy of Science 2, 49-55.

Mehetre, S.S., Mahajan, C.R., Patil, P.A., Hajare, D.N., 1994. Genetic divergence in soybean [Glycine max (L.) Merrill]. Indian Journal of Genetics and Plant Breeding 54(1), 83-88.

Rao, C.R., 1952. Advance statistical methods in biometrical research. John Wiley and Sons, New York, 383.

Shrivastava, A.N., Shrivastava, M.K., Manjaiya, J.G., 2011. Significance of four seeded pod trait in soybean yield improvement. Soybean Research 9, 53-61.

Sichkar, V.I., Grigoryan, E.M., Lugavoi, A.P., 1988. Multivariate analysis of Mahalanobis distance parameters for economically useful characters in different groups of soybean. Genetica 22, 37-43.

Singh, R.K., Chaudhary, B.D., 1979. Biometrical methods in quantitative genetic analysis. Kalyani Publication, New Delhi, 120.

Tyagi, S.D., Khan, M.H., Tyagi, V., 2012. Genetic Divergence in Soybean [Glycine max (L.) Merrill] grown in over environments. Indian Journal of Ecology 39(2), 272-275.

Zafar, I., Arshad, M., Ashraf, M., Naeem, R.M.F., Waheet, A., 2010. Genetic divergence and correlation studies of soybean [Glycine max (L.) Merrill.] genotypes. Pakistan Journal of Botany 42(2), 971-976. 